\title{
Hospital end-of-life care in haematological malignancies
}

\section{Yvan Beaussant, ${ }^{1,2}$ Etienne Daguindau, ${ }^{3}$ Adrien Chauchet, ${ }^{3}$ Philippe Rochigneux, ${ }^{4}$ Christophe Tournigand, ${ }^{5,6}$ Régis Aubry, ${ }^{1,2}$ Lucas Morin ${ }^{7}$}

- Additional material is published online only. To view, please visit the journal online (http://dx.doi.org/10.1136/ bmjspcare-2017-001446).

'Department of Palliative Care, Besancon University Hospital, Besançon, France ${ }^{2}$ Inserm CIT808, Besancon University Hospital, Besancon, France

${ }^{3}$ Hematology Department, Besancon University Hospital, Besancon, France ${ }^{4}$ Department of Medical Oncology, Paoli-Calmettes Cancer Institute, Marseille, France

${ }^{5}$ Department of Medical Oncology, Hopital Henri Mondor, Assistance Publique-Hôpitaux de Paris, Créteil, France ${ }^{6}$ Paris-Est Creteil University, Créteil, France

${ }^{7}$ Aging Research Center, Karolinska Institutet, Stockholm, Sweden

\section{Correspondence to} Lucas Morin, Aging Research Center, Karolinska Institutet, Stockholm 113 30, Sweden; lucas.morin@ki.se

Received 10 October 2017 Revised 18 January 2018 Accepted 24 January 2018 Published Online First 6 February 2018

\section{Check for updates}

To cite: Beaussant $Y$, Daguindau E, Chauchet A, et al. BMJ Supportive \& Palliative Care 2018;8:314-324.

\begin{abstract}
Objective To investigate patterns of care during the last months of life of hospitalised patients who died from different haematological malignancies. Methods Nationwide register-based study, including all hospitalised adults $\geq 20$ years who died from haematological malignancies in France in 2010-2013. Outcomes included use of invasive cancer treatments and referral to palliative care. Percentages are adjusted for sex and age using direct standardisation.
\end{abstract}

Results Of 46629 inpatients who died with haematological malignancies, $24.5 \%$ received chemotherapy during the last month before death, $48.5 \%$ received blood transfusion, $12.3 \%$ were under invasive ventilation and $18.1 \%$ died in intensive care units. We found important variations between haematological malignancies. The use of chemotherapy during the last month of life varied from $8.6 \%$ among patients with chronic myeloid leukaemia up to $30.1 \%$ among those with non-Hodgkin's lymphoma $(\mathrm{P}<0.001)$. Invasive ventilation was used in $10.2 \%$ of patients with acute leukaemia but in $19.0 \%$ of patients with Hodgkin's lymphoma $(\mathrm{P}<0.001)$. Palliative status was reported 30 days before death in only $14.8 \%$ of patients, and at time of death in $46.9 \%$ of cases. Overall, $5.5 \%$ of haematology patients died in palliative care units.

Conclusion A high proportion of patients who died from haematological malignancies receive specific treatments near the end of life. There is a need for a better and earlier integration of the palliative care approach in the standard practice of haematology. However, substantial variation according to the type of haematological malignancy suggests that the patients should not be considered as one homogeneous group. Implementation of palliative care should account for differences across haematological malignancies.

\section{INTRODUCTION}

The treatment of haematological malignancies has made considerable progress during the last two decades. ${ }^{1} 2$ Novel targeted therapies and enhanced haematopoietic stem cell transplantation procedures have significantly improved survival in patients with lymphoma, multiple myeloma and acute leukaemia. ${ }^{3}$ However, haematological malignancies remain incurable in most situations and are still associated with high mortality. ${ }^{4}$

The palliative care needs of patients with advanced haematological malignancies have been widely acknowledged. $^{5} \quad{ }^{6}$ Decision-making and communication between physicians and patients also become more complex as the disease is progressing. ${ }^{7}$ Addressing the need for palliative and supportive care of patients with haematological malignancies could improve their quality of life and the quality of care received near the end of life. ${ }^{8}$ Yet, patients with haematological malignancies have been found to be less frequently referred to palliative care services and to receive more aggressive treatments near the end of life than patients with solid tumours. ${ }^{9-11}$ Some authors have however questioned the relevance and applicability of the quality criteria developed in the field of solid oncology for patients with haematological malignancies. $^{12-15}$ These patients may indeed present specific needs that could require other standards to assess the quality of end-of-life care. Furthermore, most studies investigating the aggressiveness of end-of-life care considered solid tumours separately (ie, distinguishing between different location of histological types) but reported haematological malignancies as one homogeneous group of diseases even though haematological malignancies encompass a wide range of clinically different situations. ${ }^{10}$

This study aimed to compare the patterns of care over the course of the last 
3 months of life of hospitalised patients who died from different haematological malignancies. We hypothesised that the provision of specific treatments and that the use of palliative care facilities varied significantly from one haematological malignancy to another.

\section{METHODS}

\section{Study design and population}

Nationwide, mortality follow-back cohort using data from the French national hospital register. This register collects administrative and medical information for all inpatient admissions and outpatient visits in France. Diagnoses are coded according to the International Classification of Diseases, 10th revision (ICD-10). All hospitalised adults ( $\geq 20$ years) who died from haematological malignancies (ICD-10 codes C81 to C95 and D46) in France between 1 January 2010 and 31 December 2013 were included. Patients were excluded from the study population if they had a concomitant solid malignancy, or had overlapping or unspecified haematological malignancies.

\section{Care outcomes}

Hospital admissions

Mean number of hospital admissions, mean number of days of hospitalisation, frequency of intensive care unit (ICU) admissions, proportion of patients who remained hospitalised continuously and number of emergency department (ED) visits were calculated for the 3-month period before death. We also examined the origin of the last hospital admission before death and the final place of death of haematology patients.

\section{Medical interventions}

We primarily investigated the administration of chemotherapy delivered in hospital (intravenously or orally), blood transfusion, invasive ventilation and haemodynamic support (ie, use of dopamine, dobutamine, dopexamine, epinephrine, norepinephrine or intravenous volume expander) within the last 3 months, 1 month and 2 weeks before death. We also reported five secondary outcomes: use of radiation therapy, artificial nutritional support (regardless of the route of administration), cardiopulmonary resuscitation, tracheal intubation and dialysis by either haemodialysis, peritoneal dialysis, hemofiltration or haemodiafiltration. These medical interventions were captured if they were performed during a hospitalisation, an outpatient visit or via a hospital-at-home service. Care outcomes were chosen based on previously published studies. ${ }^{16} 17$

\section{Reporting of palliative status and palliative care referral}

We first examined the reporting of the palliative status of patients during the last 3 months of life. This was defined as the period of time when the ICD-10 code Z51.5 'palliative care' was first mentioned in the patients' medical records (as either principal or associated diagnosis). In France, this diagnosis code is used to indicate the need for care and treatments directly related to the palliative nature of an advanced and potentially life-threatening health condition. Reporting of this diagnosis is required for activity-based payment of palliative care, and increases the value of diagnosis-related groups (DRG) used by the National Health Insurance to calculate the reimbursement of hospital stays. It is also used for enabling the patients to access certain community-based services. Therefore, the coding of palliative status is expected to be of high quality near the end of life. Second, we investigated the referral to palliative care inpatient units (ie, inpatient units entirely dedicated to the practice of specialist palliative care) or palliative care support beds (ie, beds typically grouped in two-bed to four-bed clusters and embedded in hospital departments whose medical specialty leads to caring for seriously ill patients). ${ }^{18}$ Referral to specialist palliative care was identified in hospital discharge reports, using both diagnosis-related groups and identification of medical units. Details are available from the authors on request.

\section{Definition of covariates}

Sex, age (categorised into 10-year groups in order to maintain anonymity) and number of other chronic conditions (derived from the list of chronic diseases included in the Charlson Comorbidity Index ${ }^{19}$ and computed from inpatient diagnoses in the last 3 months of life) were extracted from the hospital register. Haematological conditions were categorised into 'acute leukaemia', 'chronic lymphoid leukaemia', 'chronic myeloid leukaemia', 'Hodgkin's lymphoma', 'multiple myeloma', 'myelodysplastic syndromes' and 'non-Hodgkin's lymphoma'. Details about the corresponding ICD-10 codes are available in online supplementary table A1.

\section{Statistical analysis}

Care outcomes of patients with different haematological malignancies were standardised for both sex and age, using direct standardisation method with the total population as reference. This allowed for removing the confounding effect of sex and age. Differences were tested with Pearson's $X^{2}$ test, Student's t-test and Wilcoxon rank sum test when appropriate (two-sided $\mathrm{P}$, with $\mathrm{P}<0.001)$. Multivariable logistic regression analyses were also performed to investigate the association between the type of haematological malignancy and the receipt of specific treatments while adjusting for sex, age, number of other chronic conditions and category of hospital were death occurred. Adjusted ORs were reported with their 95\% CI. All statistical analyses were performed using SAS JMP V.12.1.0 (SAS Institute). 


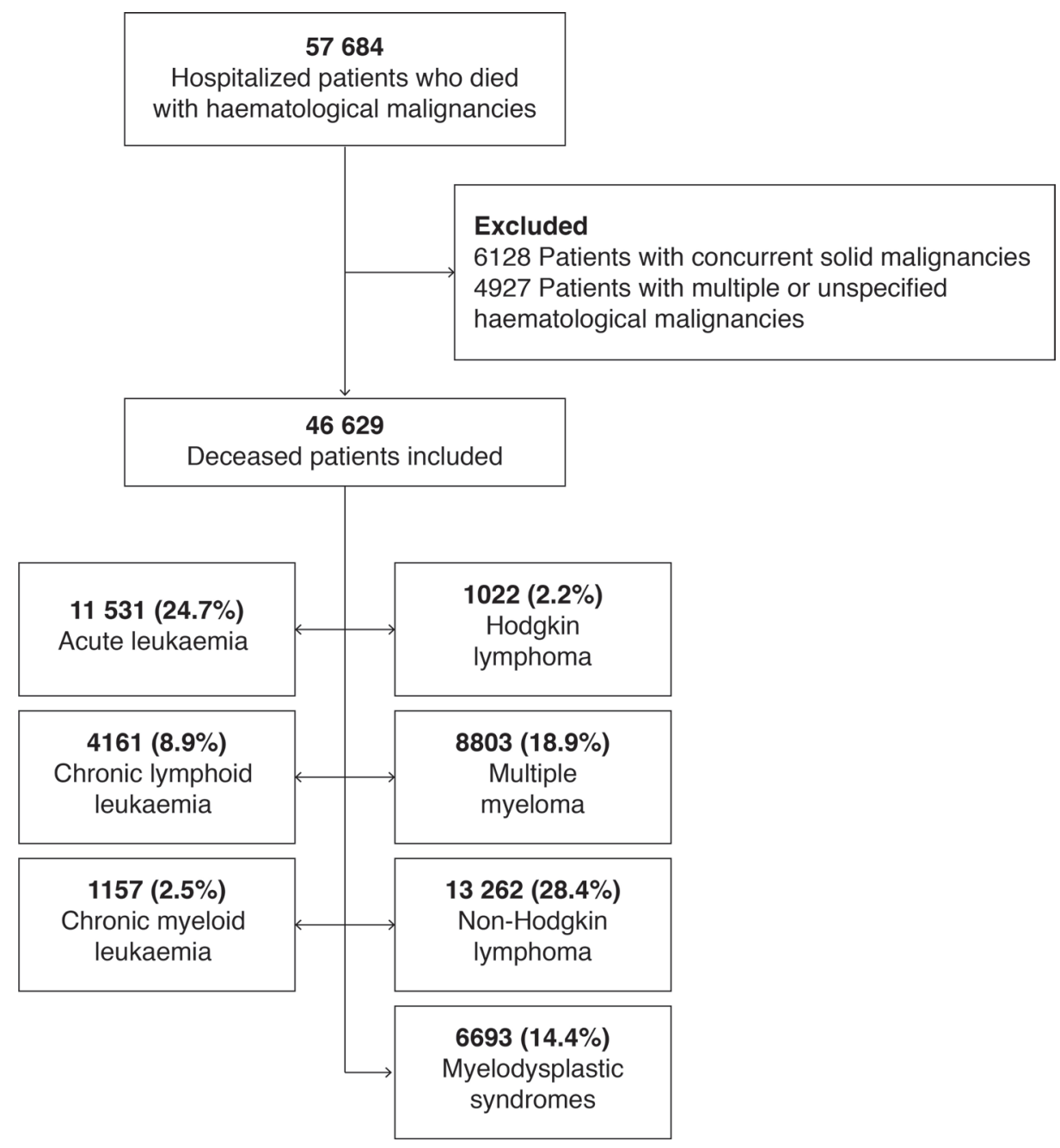

Figure 1 Selection of study population flow chart.

\section{RESULTS}

\section{Patients characteristics}

A total of 46629 patients with haematological malignancies who died in hospitals between 2010 and 2013 met our inclusion criteria (figure 1). Most patients suffered from non-Hodgkin's lymphoma (28.4\%), acute leukaemia (24.7\%) or multiple myeloma (18.9\%). Overall, $74.3 \%$ of individuals were aged $\geq 70$ years at time of death. This proportion, however, ranged from $50.5 \%$ in patients with Hodgkin's lymphoma to $90.4 \%$ in those with myelodysplastic syndromes (table 1). Overall, $79.5 \%$ of patients died in public hospitals (including 28\% in university hospitals), and 3.4\% died in comprehensive cancer centres. The proportion of patients who had no other reported chronic condition varied from $24.0 \%$ in patients with myelodysplastic syndromes to $52.0 \%$ in those with acute leukaemia, with an average number of comorbidities ranging from 1.5 to 0.7 , respectively (mean difference $=-0.72,95 \%$ CI -0.75 to -0.69$)$. The most prevalent conditions were heart failure $(25.8 \%)$, renal failure $(16.2 \%)$ and diabetes (15.6\%). Detailed information regarding the prevalence of comorbidities according to the type of haematological malignancy is available in online supplementary appendix table A2.

\section{Hospitalisations in the last $\mathbf{3}$ months of life}

During the last 3 months before death, patients had on average 2.1 hospitalisations $(\mathrm{SD}=1.6)$, and were hospitalised for 34.9 days $(\mathrm{SD}=25.5)$. Overall, $3.2 \%$ of all 46629 patients remained hospitalised continuously. As shown in table 2, this proportion varied from $1.8 \%$ of patients with acute leukaemia to $5.2 \%$ of patients with chronic myeloid leukaemia. Moreover, $58.4 \%(n=27231 / 46629)$ of patients had at least one ED visit. While adjusting for possible confounders, patients with myelodysplastic syndromes were more likely to have multiple ED visits than patients with Hodgkin's lymphoma (OR 1.45, 95\% CI 1.19 to 1.78), multiple myeloma (OR 1.40, 95\% CI 1.29 to 1.53 ) or non-Hodgkin's lymphoma (OR 1.39, 95\% CI 1.28 to 1.51 ). The place of death also varied according to the type of haematological malignancy (table 2). Compared with patients with acute leukaemia, those with Hodgkin's lymphoma were more likely to have died in intensive care units (OR 1.85, 95\% CI 1.59 


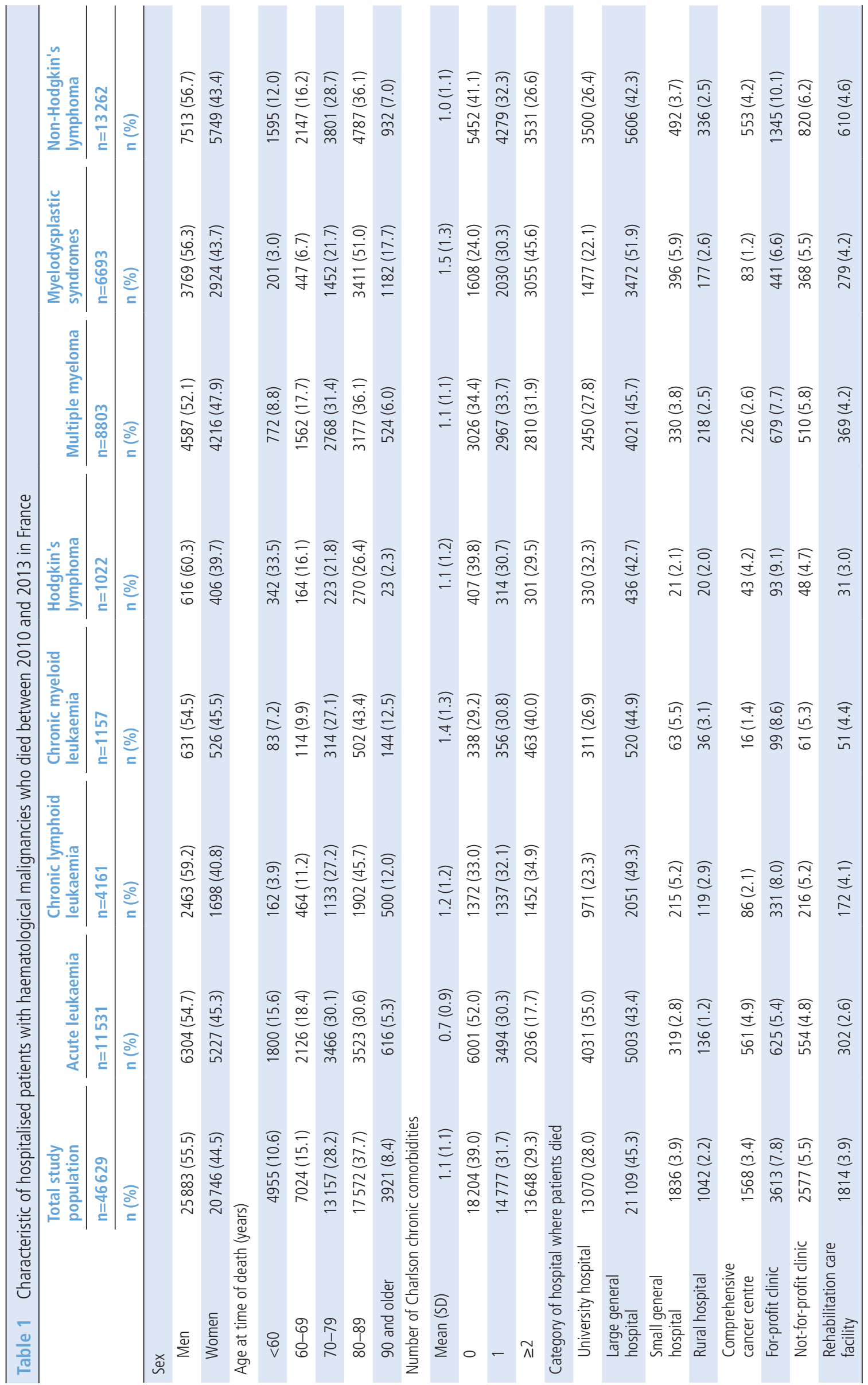




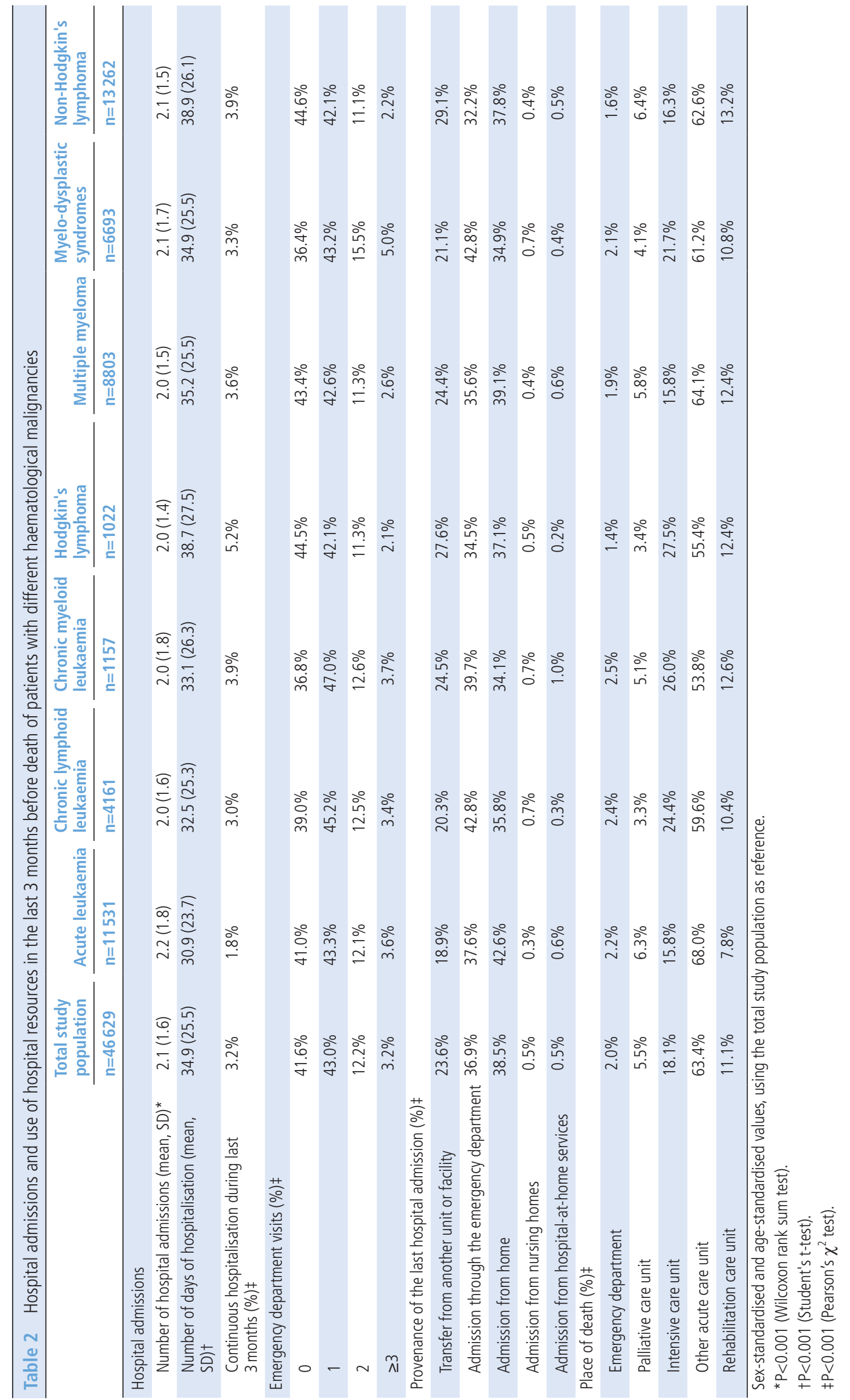


to 2.15), and less likely to have died in palliative care units (OR 0.61; 95\% CI 0.42 to 0.85 ).

\section{Treatment use over the course of the last 3 months of life} Overall, out of a total of 46629 inpatients, 17655 (37.9\%) received chemotherapy over the course of the last 3 months before death, 26701 (57.3\%) received blood transfusion, $5970(12.8 \%)$ received invasive ventilation and 7053 (15.1\%) received haemodynamic support. We found considerable variation in the use of these treatments, both over time and between haematological malignancies (see online supplementary appendix figure A1 and table A3 and Table A3). During the last month before death, the use of chemotherapy varied from $8.6 \%$ of patients with chronic myeloid leukaemia to $30.1 \%$ of patients with non-Hodgkin's lymphoma, while the use of invasive ventilation varied from $10.2 \%$ of patients with acute leukaemia to $19.0 \%$ of patients with Hodgkin's lymphoma (table 3). Likewise, although $41.7 \%$ of all patients received blood transfusion within the last 2 weeks before death, this proportion ranged from $28.3 \%$ of patients with non-Hodgkin's lymphoma to $59 \%$ of patients with acute leukaemia $(\mathrm{P}<0.001)$. These variations remained while adjusting for potential confounders (table 4). Although less frequent, artificial nutrition, tracheotomy and dialysis were still common during the last month of life.

\section{Palliative care referral to in the last 3 months of life}

The reporting of palliative status gradually increased over the last 3 months before death, from $14.8 \%$ of patients 30 days before death to $46.9 \%$ at time of death. However, $53.1 \%$ of patients died without any reporting of their palliative status. This proportion was especially high for patients with myelodysplastic syndromes, chronic lymphoid leukaemia and chronic myeloid leukaemia $(64.4 \%, 63.5 \%$ and $61.4 \%$, respectively). Also, $6 \%$ of patients were admitted in palliative care units during their last 3 months of life, and 5.5\% eventually died in palliative care units. We found only little variation according to the type of haematological malignancy (table 5), but considerable discrepancies across hospital facilities. The proportion of deaths occurring in palliative care units ranged from $0 \%$ in comprehensive cancer centres (0/1559) to $32.5 \%$ (728/1502) in private-not-for-profit clinics.

\section{DISCUSSION}

Our study shows high rates of treatment use in the last months of life of hospitalised patients with haematological malignancies. Overall, $25 \%$ of decedents received chemotherapy during the last month before death, $48 \%$ received blood transfusion, $12 \%$ had invasive ventilation and $18 \%$ died in ICUs. Furthermore, the use of invasive ventilation and haemodynamic support increased steadily during the last 3 months of life.
Patients with Hodgkin's lymphoma or acute leukaemia died younger and had less comorbidities than patients with other haematological malignancies. These patients were also more likely to die in tertiary hospitals than patients who died from chronic lymphoid leukaemia or myelodysplastic syndromes. This may be due to the necessity for patients with Hodgkin's disease or acute leukaemia to have access to highly specialised physicians and treatments. In addition, the overall prevalence of chemotherapy administration during the last month before death varied from $<10 \%$ in patients with chronic myeloid leukaemia or myelodysplastic syndromes up to $30 \%$ in patients who died from non-Hodgkin's lymphoma. Clinical factors most likely explain a large share of this variation: haematological malignancies have different levels of chemosensitivity, are more or less likely to induce severe cytopenia, have different prognoses and shape different end-of-life trajectories in terms of functional decline and psychological distress. In our opinion, these variations could also explain the considerable heterogeneity in the findings reported in previous studies, with rates of chemotherapy use in the last month of life ranging from $14 \%$ to $43 \% .^{9}{ }^{20}$

Studies investigating ICU admission of haematology patients also showed contrasted results, with $19 \%-33 \%$ of patients dying in ICU. ${ }^{21}$ Our results show important variations between the different haematological malignancies (from $15 \%$ to $27 \%$ ), thus suggesting disease-specific patterns of ICU referral near the end of life. For instance, it could be hypothesised that because of their higher survival rates, patients with Hodgkin's lymphoma or chronic myeloid leukaemia were more likely to benefit from ICU care than patients with other haematological malignancies. However, these high rates of patients who died in ICU are a matter of concern, given that patients who die in ICU often experience greater physical and emotional distress and worse quality of life than patients who die at home with hospice care, and that their caregivers may have a higher risk of prolonged grief. ${ }^{22}$ Decisions to transfer patients with advanced haematological malignancies to ICUs should therefore be anticipated and carefully examined. ${ }^{23} 24$

Blood transfusion near the end of life is a critical issue in haematology, and is frequently continued close to death even in palliative care services. ${ }^{25}$ Classifying it as an 'aggressive' treatment is debatable. Because bone marrow failure is frequent in haematological malignancies, blood transfusion represents an early life-supporting intervention, often associated with brisk clinical improvement. At the end of life, these treatments might have been considered as a tool to improve the quality of life. ${ }^{2627}$ On the other hand, blood transfusion may hinder death at home or in hospice, and contribute to prolong patients' lives beyond their wish. ${ }^{2829}$ Thus, the decision to continue or discontinue blood transfusion near the end of life should take into 


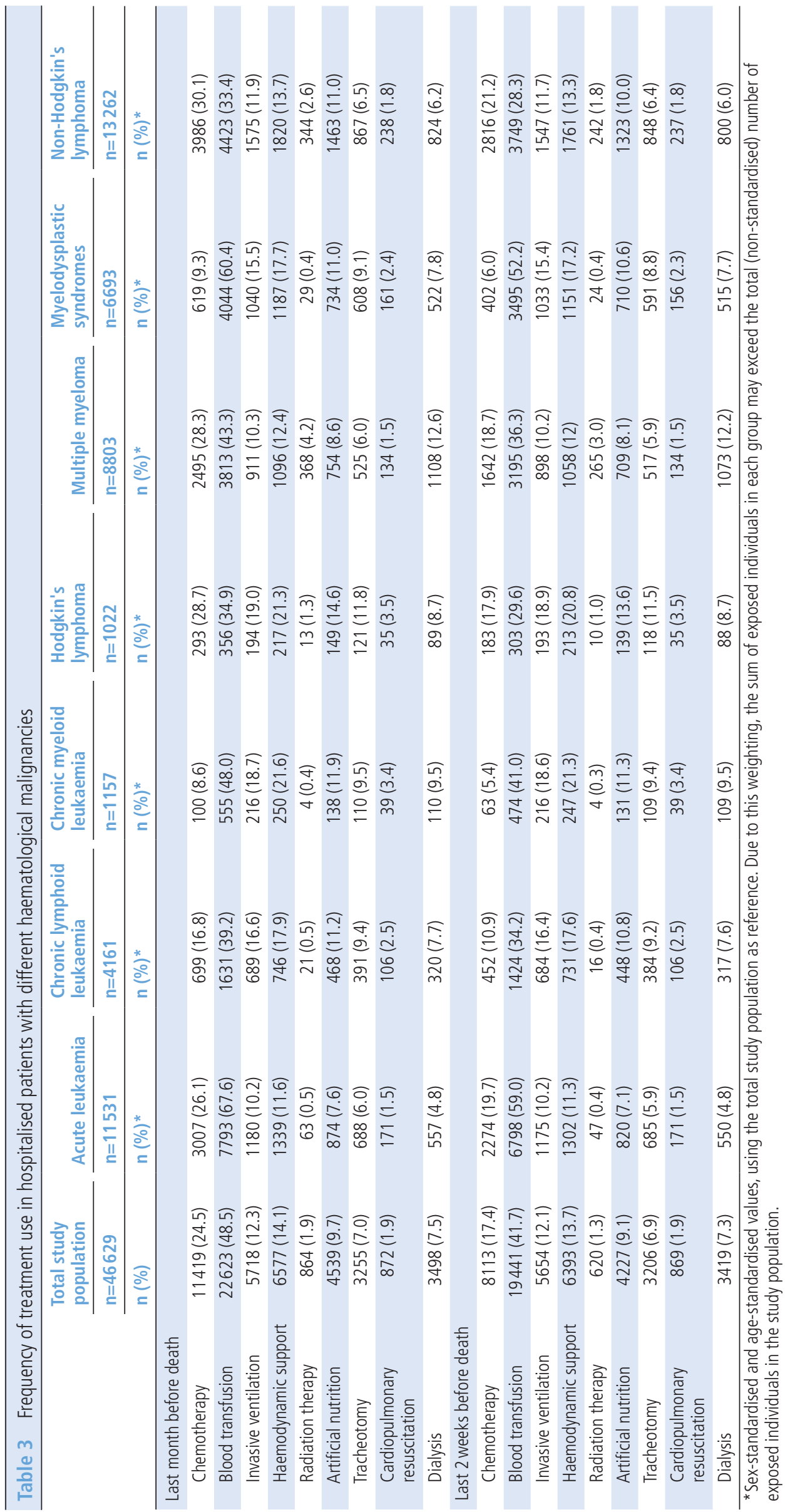


Table 4 Adjusted likelihood for hospitalised patients with different haematological malignancies to receive specific treatments during the last month before death

\begin{tabular}{|c|c|c|c|c|}
\hline & Chemotherapy & Blood transfusion & Invasive ventilation & Haemodynamic support \\
\hline & Adjusted OR $(95 \% \mathrm{CI})$ * & Adjusted OR $(95 \% \mathrm{CI})^{*}$ & Adjusted OR $(95 \% \mathrm{CI})^{*}$ & Adjusted OR $(95 \% \mathrm{CI})^{*}$ \\
\hline Acute leukaemia & 1 & 1 & 1 & 1 \\
\hline Chronic lymphoid leukaemia & 0.57 (0.51 to 0.63$)$ & $0.30(0.28$ to 0.33$)$ & 1.81 (1.62 to 2.03$)$ & $1.70(1.53$ to 1.90$)$ \\
\hline Chronic myeloid leukaemia & $0.28(0.22$ to 0.34$)$ & $0.48(0.42$ to 0.54$)$ & 1.97 (1.63 to 2.36$)$ & 1.99 (1.68 to 2.36$)$ \\
\hline Hodgkin's disease & $1.04(0.90$ to 1.21$)$ & 0.28 (0.25 to 0.32$)$ & 1.93 (1.64 to 2.28$)$ & 1.99 (1.69 to 2.33$)$ \\
\hline Multiple myeloma & 1.19 (1.12 to 1.27$)$ & $0.38(0.36$ to 0.40$)$ & $0.96(0.87$ to 1.05$)$ & 1.03 (0.94 to 1.12$)$ \\
\hline Myelodysplastic syndromes & $0.24(0.22$ to 0.27$)$ & 0.81 (0.76 to 0.87$)$ & 1.37 (1.22 to 1.52$)$ & 1.39 (1.25 to 1.53$)$ \\
\hline Non-Hodgkin's lymphoma & $1.29(1.22$ to 1.37$)$ & $0.23(0.22$ to 0.25$)$ & $1.19(1.10$ to 1.30$)$ & $1.22(1.13$ to 1.32$)$ \\
\hline
\end{tabular}

*ORs were calculated by the mean of logistic regression models adjusted for sex, age, number of Charlson comorbidities and category of hospital were death occurred. Acute leukaemia was chosen as the reference category.

account both the patient's needs in terms of comfort, the effectiveness of such treatments and the existence of other possible alternatives. ${ }^{30}$

Palliative status of patients remained unreported for more than half of individuals who died from haematological malignancies, and was often reported late during the course of the disease: only $14.8 \%$ of patients had a palliative status identified 1 month before death. Surprisingly, patients with myelodysplastic syndromes and chronic lymphoid leukaemiawhose goals of care are mostly palliative-were the least likely to have a palliative status reported in their electronic medical records. This is consistent with recent findings from Odejide et al, who suggested a less frequent use of hospice among patients who died from indolent lymphomas compared with those who died from aggressive lymphomas. ${ }^{10}$ Other studies have highlighted variations in palliative care referrals depending on the type of haematological malignancy, with heterogeneous results. ${ }^{31} 32$ In France, 130 palliative care inpatient units are specifically dedicated to patients whose clinical, psychosocial and ethical situation are the most complex. Given the high physical and psychological burden of patients dying from haematological malignancies, the low rate of palliative care unit referral is unexpected and raises concern. Palliative care referral has indeed been found to be associated with a decreased aggressiveness of end-oflife care and with an increased quality of life and satisfaction with care. ${ }^{33} 34$ Our results are however consistent with previous studies, showing that referral to specialist palliative care services for haematology patients remains rare and late in the disease trajectory. ${ }^{35}$ Despite the unavoidable uncertainty attached to the prediction of the remaining life-expectancy, overall survival in refractory haematological malignancies rarely exceeds a few months. For these patients, palliative care referral should therefore occur even when further active treatments are considered. ${ }^{36}$

Several factors are likely to contribute to the aggressiveness of end-of-life care, including the overoptimism induced by the myriad of treatment options, the unpredictable trajectory of advanced haematological malignancies and the sense of failure often perceived by haematologists facing refractory diseases. ${ }^{3738}$ These factors tend to meet patients' need for hope as well as -in many instances-their desire for a passive role in the decision-making. ${ }^{39}$ Qualitative studies have described this phenomenon of patients and physicians mutually reinforcing attitudes of 'not giving up' ${ }^{70}$ The insufficient integration of a palliative care approach in the standard practice of haematology and the lack of timely end-of-life discussions with patients are also a major issue for improving quality of end-oflife care for patients dying from haematological malignancies. ${ }^{64142}$

On the other hand, healthcare providers should better integrate the needs of haematology patients in the delivery of specialist and generalist palliative care. The various outcomes developed to assess the quality of end-of-life care in patients dying from solid cancer can not be transposed de facto to patients dying from haematological malignancies; and even less so when the latter are considered as a homogeneous whole. Haematology patients often experience bleeding episodes, severe or unusual infections and specific medications (eg, immunosuppressive drugs) that may lead GPs and other generalist physicians to request the transfer of these patients to specialised acute units. Clinical haematologists consulted in focus groups therefore stated that current quality measures were hardly acceptable for patients with haematological malignancies, and that other indicators would reflect more accurately the aggressiveness of care near the end of life. ${ }^{11}$ In a qualitative interview-based study of 45 British clinicians involved in end-of-life care for patients with haematological malignancies, researchers sought to understand the reasons that could explain the high proportion of patients dying in hospitals. ${ }^{43}$ Their findings suggest that prognostic uncertainty, unpredictable illness trajectories, and difficulties in identifying when to withdraw active treatments play an important role. 


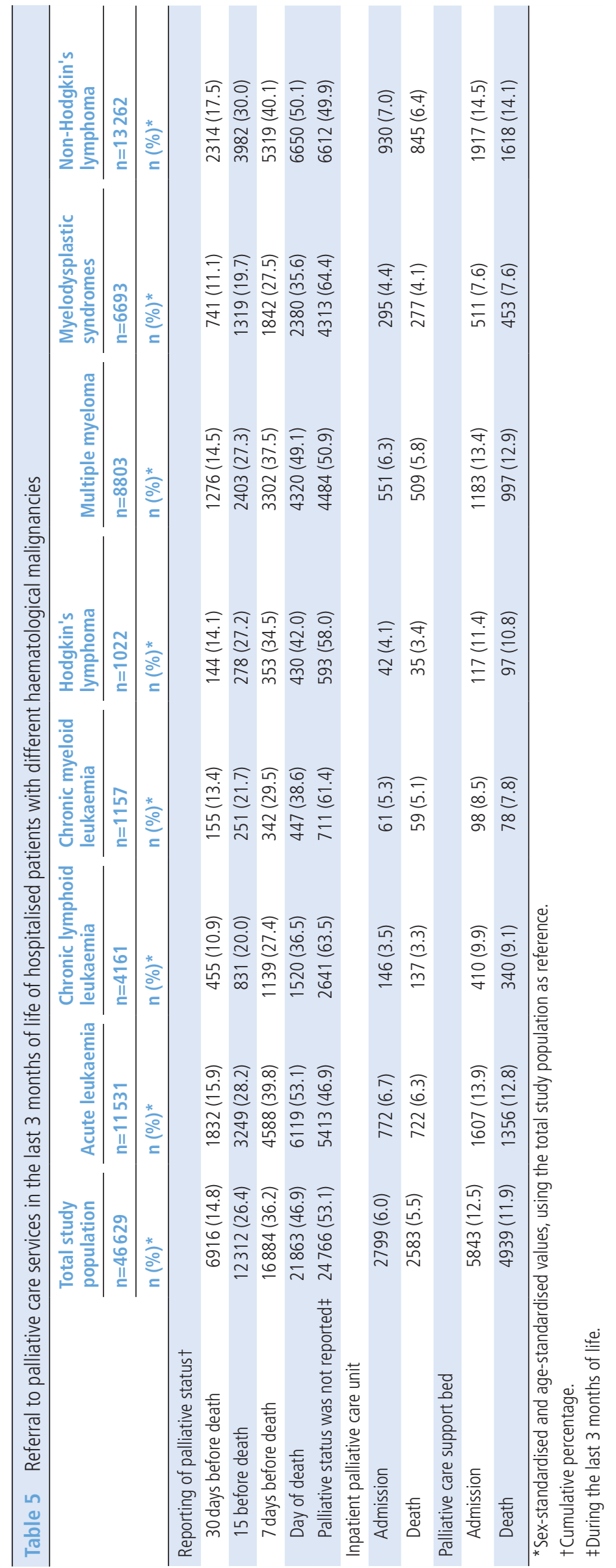


Our findings should be interpreted in light of several limitations. First, data collection was based on a national hospital register, and therefore gives access only to hospital records and not to community-based care providers or nursing home facilities data. Since in-hospital death is often considered as indicative of a form of aggressive care itself, our study focused on a selected population that was more likely to receive aggressive treatments. Nevertheless, in France about $75 \%-80 \%$ of patients with haematological malignancies die in hospitals, and these findings cover the entire country with no selection of the patients based on their healthcare insurance scheme or their affiliation to a specific care provider. It should also be noted that oral molecular targeted therapy or immunotherapy delivered in community pharmacies were not reported in our dataset, which may underestimate the actual use of chemotherapy near the end of life. Second, we relied on ICD-10 diagnosis codes to identify primary and secondary malignancies: since we could not confirm these diagnoses with histological or imaging-based data, we cannot exclude inaccuracies in the characterisation of cancers. Third, we had no access to the date of diagnosis, the disease status, the type and number of disease-directed agents or quality-of-life measures. Furthermore, we had no information regarding surgical procedures and haematopoietic stem cell transplant. Fourth, we based our assessment of the timing of palliative status reporting on ICD-10 coding (Z51.5). This could lead to under-reporting of the actual proportion of patients whose situation was in fact identified and managed as palliative. Finally, our findings may not be entirely generalisable to other healthcare systems or countries.

\section{CONCLUSION}

This article reports the first population-level study comparing the patterns of end-of-life care between different haematological malignancies. Consistently with previous reports, we found high rates of treatment use and low rates of palliative care referral. However, we also found substantial variation according to the type of haematological malignancy. Future studies should therefore differentiate haematological malignancies in the same manner as they differentiate solid tumours. These findings suggest the need for a better and earlier integration of the palliative care approach in the standard practice of haematology, while taking into account the specific needs of patients with advanced haematological malignancies.

Acknowledgements The authors acknowledge the contribution of the French Technical Agency for Hospital Information (ATIH) during the extraction of the data presented in this article.

Contributors LM had full access to all of the data in the study and takes responsibility for the integrity of the data and the accuracy of the data analysis. Study concept and design: LM, YB, AC, ED, PR. Acquisition of data: LM, RA, CT. Statistical analysis: LM. Interpretation of data: all authors. Drafting of manuscript or revision for important intellectual content: all authors.

Competing interests None declared.

Ethics approval This study was reviewed and approved by the French National Data protection Agency (CNIL, decision $n^{\circ}$ DE-2014-112). In accordance with French regulation, additional ethical permit was not required because of the nature of the data collection (non-interventional study using irreversibly de-identified data related to deceased individuals).

Provenance and peer review Not commissioned; externally peer reviewed.

Data sharing statement Raw data cannot be made publicly available because of privacy issues. Additional tables can be provided by the authors on reasonable request.

(C) Article author(s) (or their employer(s) unless otherwise stated in the text of the article) 2018. All rights reserved. No commercial use is permitted unless otherwise expressly granted.

\section{REFERENCES}

1 Bower H, Björkholm M, Dickman PW, et al. Life expectancy of patients with chronic myeloid leukemia approaches the life expectancy of the general population. J Clin Oncol 2016;34:2851-7.

2 Brenner H, Gondos A, Pulte D. Trends in long-term survival of patients with chronic lymphocytic leukemia from the 1980s to the early 21st century. Blood 2008;111:4916-21.

3 Gooley TA, Chien JW, Pergam SA, et al. Reduced mortality after allogeneic hematopoietic-cell transplantation. $\mathrm{N} \mathrm{Engl} \mathrm{J}$ Med 2010;363:2091-101.

4 Sant M, Minicozzi P, Mounier M, et al. Survival for haematological malignancies in Europe between 1997 and 2008 by region and age: results of EUROCARE-5, a population-based study. Lancet Oncol 2014;15:931-42.

5 LeBlanc TW, El-Jawahri A. When and why should patients with hematologic malignancies see a palliative care specialist? Hematology Am Soc Hematol Educ Program 2015;2015:471-8.

6 Zimmermann C. Palliative care for patients with hematological malignancies: time for a new model. Leuk Res 2016;48:78-9.

7 Beaussant Y, Mathieu-Nicot F, Pazart L, et al. Is shared decision-making vanishing at the end-of-life? A descriptive and qualitative study of advanced cancer patients' involvement in specific therapies decision-making. BMC Palliat Care $2015 ; 14: 61$.

8 Hui D, Kim SH, Roquemore J, et al. Impact of timing and setting of palliative care referral on quality of end-of-life care in cancer patients. Cancer 2014:120:1743-9.

9 Hui D, Didwaniya N, Vidal M, et al. Quality of end-of-life care in patients with hematologic malignancies: a retrospective cohort study. Cancer 2014;120:1572-8.

10 Odejide OO, Cronin AM, Earle CC, et al. Hospice use among patients with lymphoma: impact of disease aggressiveness and curability. J Natl Cancer Inst 2016;108.

11 Odejide OO, Cronin AM, Condron NB, et al. Barriers to quality end-of-life care for patients with blood cancers. J Clin Oncol 2016;34:3126-32.

12 LeBlanc TW, Abernethy AP, Casarett DJ. What is different about patients with hematologic malignancies? A retrospective cohort study of cancer patients referred to a hospice research network. J Pain Symptom Manage 2015;49:505-12.

13 Morikawa M, Shirai Y, Ochiai R, et al. Barriers to the collaboration between hematologists and palliative care teams on relapse or refractory leukemia and malignant lymphoma patients' care: a qualitative study. Am J Hosp Palliat Care 2016;33:977-84. 
14 Odejide OO, Cronin AM, Condron N, et al. Timeliness of end-of-life discussions for blood cancers: a national survey of hematologic oncologists. JAMA Intern Med 2016;176:263-5.

15 Odejide OO, Salas Coronado DY, Watts CD, et al. End-oflife care for blood cancers: a series of focus groups with hematologic oncologists. J Oncol Pract 2014;10:e396-e403.

16 Earle CC, Landrum MB, Souza JM, et al. Aggressiveness of cancer care near the end of life: is it a quality-of-care issue? J Clin Oncol 2008;26:3860-6.

17 Bekelman JE, Halpern SD, Blankart CR, et al. Comparison of site of death, health care utilization, and hospital expenditures for patients dying with cancer in 7 developed countries. JAMA 2016;315:272-83.

18 Morin L, Aubry R. The development of palliative care services in France (1987-2013). Médecine Palliat 2015;14:134-41.

19 Quan H, Li B, Couris CM, et al. Updating and validating the Charlson comorbidity index and score for risk adjustment in hospital discharge abstracts using data from 6 countries. Am J Epidemiol 2011;173:676-82.

20 Emanuel EJ, Young-Xu Y, Levinsky NG, et al. Chemotherapy use among Medicare beneficiaries at the end of life. Ann Intern Med 2003;138:639-43.

21 Brück P, Pierzchlewska M, Kaluzna-Oleksy M, et al. Dying of hematologic patients--treatment characteristics in a German University Hospital. Support Care Cancer 2012;20:2895-902.

22 Wright AA, Keating NL, Balboni TA, et al. Place of death: correlations with quality of life of patients with cancer and predictors of bereaved caregivers' mental health. J Clin Oncol 2010;28:4457-64.

23 Hill QA. Intensify, resuscitate or palliate: decision making in the critically ill patient with haematological malignancy. Blood Rev 2010;24:17-25.

24 Azoulay E, Mokart D, Pène F, et al. Outcomes of critically ill patients with hematologic malignancies: Prospective multicenter data from France and Belgium. J Clin Oncol 2013;31:2810-8.

25 Alt-Epping B, Wulf G, Nauck F. Palliative care for patients with hematological malignancies-a case series. Ann Hematol 2011;90:613-5.

26 Monti M, Castellani L, Berlusconi A, et al. Use of red blood cell transfusions in terminally ill cancer patients admitted to a palliative care unit. J Pain Symptom Manage 1996;12:18-22.

27 Uceda Torres ME, Rodríguez Rodríguez JN, Sánchez Ramos $\mathrm{JL}$, et al. Transfusion in palliative cancer patients: a review of the literature. J Palliat Med 2014;17:88-104.

28 Preston NJ, Hurlow A, Brine J, et al. Blood transfusions for anaemia in patients with advanced cancer. Cochrane Database Syst Rev 2012;2:CD009007.

29 Mannis GN, McNey LM, Gupta NK, et al. The transfusion tether: Bridging the gap between end-stage hematologic malignancies and optimal end-of-life care. Am J Hematol 2016;91:364-5.

30 Jaulmes D, Ceccaldi J, Colombat P. Blood transfusion in the hemopathies at the end of the lifetime: ethical and organisational aspects. Hematologie 2010;16:228-34.

31 Howell DA, Wang HI, Roman E, et al. Variations in specialist palliative care referrals: findings from a population-based patient cohort of acute myeloid leukaemia, diffuse large B-cell lymphoma and myeloma. BMJ Support Palliat Care 2015;5:496-502.

32 Howell DA, Shellens R, Roman E, et al. Haematological malignancy: are patients appropriately referred for specialist palliative and hospice care? A systematic review and metaanalysis of published data. Palliat Med 2011;25:630-41.

33 Jang RW, Krzyzanowska MK, Zimmermann C, et al. Palliative care and the aggressiveness of end-of-life care in patients with advanced pancreatic cancer. J Natl Cancer Inst 2015;107:1-8.

34 Gaertner J, Siemens W, Meerpohl JJ, et al. Effect of specialist palliative care services on quality of life in adults with advanced incurable illness in hospital, hospice, or community settings: systematic review and meta-analysis. BMJ 2017;357:j2925

35 Ansell P, Howell D, Garry A, et al. What determines referral of UK patients with haematological malignancies to palliative care services? An exploratory study using hospital records. Palliat Med 2007;21:487-92.

36 Hui D, Bruera E. Integrating palliative care into the trajectory of cancer care. Nat Rev Clin Oncol 2016;13:159-71.

37 Hui D, Bansal S, Park M, et al. Differences in attitudes and beliefs toward end-of-life care between hematologic and solid tumor oncology specialists. Ann Oncol 2015;26:1440-6.

38 LeBlanc TW, O'Donnell JD, Crowley-Matoka M, et al. Perceptions of palliative care among hematologic malignancy specialists: a mixed-methods study. J Oncol Pract 2015;11:e230-e238.

39 Ernst J, Brähler E, Aldaoud A, et al. Desired and perceived participation in medical decision-making in patients with haemato-oncological diseases. Leuk Res 2010;34:390-2.

40 Buiting HM, Rurup ML, Wijsbek H, et al. Understanding provision of chemotherapy to patients with end stage cancer: qualitative interview study. Br Med J 1933.

41 Manitta VJ, Philip JA, Cole-Sinclair MF. Palliative care and the hemato-oncological patient: can we live together? A review of the literature. J Palliat Med 2010;13:1021-5.

42 LeBlanc TW, Care P. Palliative care and hematologic malignancies: old dog, new tricks? J Oncol Pract 2014;10:e404-e407.

43 McCaughan D, Roman E, Smith AG, et al. Determinants of hospital death in haematological cancers: findings from a qualitative study. BMJ Support Palliat Care 2018;8:78-86. 